\title{
An estimate of the magnetic field strength associated with a solar coronal mass ejection from low frequency radio observations
}

\author{
K. Sasikumar Raja ${ }^{1}$, R. Ramesh ${ }^{1}$, K. Hariharan ${ }^{1}$, C. Kathiravan ${ }^{1}$, T. J. Wang² \\ sasikumar@iiap.res.in
}

Received __; accepted _

\footnotetext{
${ }^{1}$ Indian Institute of Astrophysics, Bangalore 560034

${ }^{2}$ Department of Physics, The Catholic University of America and NASA Goddard Space Flight Center, Code 671, Greenbelt, MD 20771, USA
} 


\begin{abstract}
We report ground based, low frequency heliograph (80 MHz), spectral (85-35 $\mathrm{MHz}$ ) and polarimeter (80 and $40 \mathrm{MHz}$ ) observations of drifting, non-thermal radio continuum associated with the 'halo' coronal mass ejection (CME) that occurred in the solar atmosphere on 2013 March 15. The magnetic field strengths $(B)$ near the radio source were estimated to be $B \approx 2.2 \pm 0.4 \mathrm{G}$ at $80 \mathrm{MHz}$ and $B \approx 1.4 \pm 0.2 \mathrm{G}$ at $40 \mathrm{MHz}$. The corresponding radial distances $(r)$ are $r \approx 1.9 R_{\odot}$ $(80 \mathrm{MHz})$ and $r \approx 2.2 R_{\odot}(40 \mathrm{MHz})$.
\end{abstract}

Subject headings: Sun - activity: Sun - flares: Sun - corona: Sun - radio radiation: Sun: coronal mass ejections (CMEs): Sun - magnetic topology 


\section{Introduction}

It is known that intense, long-lasting (tens of minutes to hours) non-thermal radio continuum are observed sometimes in association with the flares and CMEs in the solar atmosphere. Boischot (1957) had designated these events as type IV bursts. Further studies showed that there are two classes of type IV bursts: The first variety occurs after the impulsive phase of the flares and drifts in the spectrum to lower frequencies. Interferometer observations indicate that the radio source exhibits outward movement through the solar atmosphere with speeds in the range $\approx 200-1500 \mathrm{~km} \mathrm{~s}^{-1}$. Emission can be observed even when the source is located at large radial distances $\left(r \approx 5 R_{\odot}\right)$ above the plasma level corresponding to the frequency of observation. The sources have low directivity and are partially circularly polarized. The sense of polarization correspond usually to the extraordinary mode (e-mode) of the magneto-ionic theory. These are called the moving type IV (type IVm) bursts. The second variety, called the stationary type IV bursts (type IVs), appear near the flare site during the impulsive phase at frequencies $\gtrsim 300 \mathrm{MHz}$ and in the post-flare phase at lower frequencies. The type IVs burst is characterized by a source whose position does not change and which is located close to or slightly above the plasma level corresponding to the frequency of observation. The emission is circularly polarized usually in the ordinary mode (o-mode) of the magneto-ionic theory. The cone of emission is narrow since type IVs bursts are rarely observed when the associated flare is near the limb of the Sun. The high directivity, location close to the plasma level, and the presence of fine structures suggest that the emission mechanism is related to the plasma frequency. The type IVs bursts at low frequencies may occur with or without a type IVm burst (Pick 1961; Boischot \& Pick 1962; Weiss 1963; Wild et al. 1963; Stewart 1985; A Aurass et al. 2005; Pick 2008).

Compared to the type IVs bursts, the type IVm are more closely associated with the 
CMEs. It is also possible to infer the CME magnetic field using them. Gergely (1986) had noted that $5 \%$ of all CMEs are associated with type IVm bursts and $33-50 \%$ of type IVm bursts are associated with CMEs. But only a few estimates of the field strength have been reported in the literature. One of the reasons for this is the non-availability of simultaneous white light and radio observations close to the Sun $\left(r \lesssim 2 R_{\odot}\right)$, and the relatively rare occurrence of the type IVm bursts White (2007). The bursts have been explained using either second harmonic plasma emission (Duncan 1981; Stewart et al. 1982b; Gopalswamy \& Kundu 1989b; Kundu et al. 1989; Ramesh et al. 2013) or optically thin non-thermal gyro-synchrotron emission (Gopalswamv \& Kundu 1989a; Bastian \& Gary 1997; Bastian et al. 2001; Maia et al. 2007; Tun \& Vourlidas 2013) in the past. Using simultaneous whitelight, radio heliograph, radio polarimeter and radio spectral observations we have presented arguments to show that the type IVm burst associated with the 'halo' CME event of 2013 March 15 can be explained on the basis of non-thermal gyro-synchrotron emission and estimated the magnetic field strength near the source region of the burst.

\section{Observations}

The radio data were obtained on 2013 March 15 at $80 \mathrm{MHz}$ with the Gauribidanur RAdioheliograPH (GRAPH; Ramesh et al. 1998; Ramesh et al. 1999a,b, 2006a) in the imaging mode, the Gauribidanur Radio Interference Polarimeter (GRIP; Ramesh et al. 2008) at $80 \mathrm{MHz}$ and $40 \mathrm{MHz}$ in the transit mode, and over the $85-35 \mathrm{MHz}$ band with the Gauribidanur LOw frequency Solar Spectrograph (GLOSS; Ebenezer et al. 2001; Ebenezer et al. 2007; Kishore et al. 2014) in the spectral mode. All the aforementioned instruments are located in the Gauribidanur radio observatory1, about $100 \mathrm{~km}$ north

\footnotetext{
${ }^{1}$ http://www.iiap.res.in/centers/radio
} 
of Bangalore in India (Ramesh 2011a). The co-ordinates of the array are Longitude = $77^{\circ} 27^{\prime} 07^{\prime \prime}$ East, and Latitude $=13^{\circ} 36^{\prime} 12^{\prime \prime}$ North. The GRAPH produces two dimensional images of the solar corona with an angular resolution of $\approx 5^{\prime} \times 7^{\prime}$ (Right Ascension, R.A. $\times$ Declination, decl.) at $80 \mathrm{MHz}$. The integration time and the bandwidth of observation are $\approx 250 \mathrm{~ms} \approx 2 \mathrm{MHz}$, respectively. GRIP is an east-west one-dimensional interferometer array and observes the circularly polarized flux density from the 'whole' Sun at $80 \mathrm{MHz}$ and $40 \mathrm{MHz}$ simultaneously. Linear polarization, if generated at the correspoding radio source region in the solar atmosphere, is presently difficult to detect at low radio frequencies because of the differential Faraday rotation of the plane of polarization within the typical observing bandwidths (Grognard \& McLean 1973). The GRIP has a broad response pattern ('beam') compared to the Sun in both R.A./east-west direction $\left(\approx 1.5^{\circ}\right.$ at $\left.80 \mathrm{MHz}\right)$ and decl./north-south direction $\left(\approx 90^{\circ}\right)$. So observations with the GRIP in the transit mode essentially reproduces its 'east-west beam' with amplitude proportional to the intensity of the emission from the 'whole' Sun, weighted by the antenna gain in the corresponding direction. The integration time and the observing bandwidth are the same as the GRAPH. The respone pattern of the GLOSS is very broad, $\approx 90^{\circ} \times 5^{\circ}$ (R.A. $\times$ decl.) and hence the Sun is a point source for the latter. The integration time and the observing bandwidth are comparatively smaller here, $\approx 100 \mathrm{~ms}$ and $\approx 300 \mathrm{kHz}$ (at each frequency), respectively. The width of the response pattern of the GLOSS in R.A. (i.e. hour angle) is nearly independent of frequency. The Sun is a point source for both the GRIP and the GLOSS. The optical data were obtained with the Large Angle and Spectrometric Coronagraph (LASCO; Brueckner et al. 1995) onboard the Solar and Heliospheric Observatory (SOHO), COR1 coronagraph of the Sun-Earth Connection Coronal and Heliospheric Investigation (SECCHI; Howard et al. 2008) onboard the Solar TErrestrial RElations Observatory (STEREO), and in $193 \AA$ with the Atmospheric Imaging Assembly (AIA; Lemen et al. 2012) onboard the Solar Dynamics Observatory (SDO). 
Figure 1 shows the dynamic spectrum obtained with the GLOSS on 2013 March 15 during the interval 06:15 - 08:15 UT in the frequency range 85-35 MHz. Two types of enhanced radio emission with differing spectral characteristics are simultaneously noticeable in the spectrum: 1 ) a weak stationary continuum during the period $\approx 06: 30$ - 08:10 UT with fine structures, and 2) a comparatively intense patch of continuum drifting from $85 \mathrm{MHz}$ to $35 \mathrm{MHz}$ during the period $\approx 06: 55-07: 50 \mathrm{UT}$. The fine structures in the background of the latter are most likely part of the ongoing stationary continuum during the same interval. The stationary and the drifting continuum described above are the typical spectral signatures of the type IVs and type IVm bursts in the solar atmosphere, respectively (Stewart 1985). The average duration $(\tau)$ of the type IVm burst in Figure 1 increases with decreasing frequency. The increase in the temporal width with decrease in frequency of the region enclosed between the 'black' lines from $85 \mathrm{MHz}$ to $35 \mathrm{MHz}$ in the spectrum indicates this. The typical widths (i.e. duration) are $\tau \approx 25 \mathrm{~min}$ and $\tau \approx 45 \mathrm{~min}$ at $85 \mathrm{MHz}$ and 35 $\mathrm{MHz}$, respectively. These are consistent with the statistical results on the duration of type IVm bursts reported by Robinson (1978). The onset of the burst at $85 \mathrm{MHz}$ is $\approx 06: 55 \mathrm{UT}$ and at $35 \mathrm{MHz}$ is $\approx 07: 05 \mathrm{UT}$.

Figure 2 shows the time profile of the Stokes I \& V radio emission from the solar corona at $80 \mathrm{MHz}$ as observed with the GRIP on 2013 March 15. Similar observations at $40 \mathrm{MHz}$ are shown in Figure 3. The observations were carried out in the transit mode and hence the observed time profile and their duration essentially corresponds to the east-west response pattern of the GRIP. The peak flux densities, estimated using the polynomial fit to the observations (see the overplotted smooth line in Figures 2 and 3 ), are $\approx 170,000$ Jy (Stokes I) and $\approx 50,000 \mathrm{Jy}$ (Stokes V) at $80 \mathrm{MHz}$, and $\approx 181,000 \mathrm{Jy}$ (Stokes I) and $\approx 62,000 \mathrm{Jy}$ (Stokes V) at $40 \mathrm{MHz}$. These values correspond mostly to that of the type IVm burst alone since we had subtracted the corresponding mean flux densities of the background type IVs burst fine structures using GRIP observations of the same outside 
the type IVm burst period. The respective values for the type IVs bursts are $\approx 110,000$ Jy (Stokes I) and $\approx 53,000 \mathrm{Jy}$ (Stokes V) at $80 \mathrm{MHz}$, and $\approx 117,000 \mathrm{Jy}$ (Stokes I) and $\approx$ 61,000 Jy (Stokes V) at $40 \mathrm{MHz}$. That the flux density of particularly the Stokes I emission for the type IVs burst is a significant fraction of that of the type IVm burst in the present case is noticeable from their contrast with respect to the background in Figure 1 also. The $80 \mathrm{MHz}$ flux densities are reasonably consistent with that reported earlier (Kail 1969). The spectral index $(\alpha)$ between $40 \mathrm{MHz}$ and $80 \mathrm{MHz}$ for the type IVm burst using the above Stokes I flux densities is $\alpha \approx-0.1$. Since the non-thermal spectral index is generally $<0$ (Kraus 1986; Subramanian \& Sastry 1988), the above value $\alpha \approx-0.1$ indicates that the observed emission in the present case is of non-thermal origin. The estimated degree of circular polarization $(d c p)$ of the type IVm burst in Figures 2 and 3 are $\approx 0.29 \pm 0.1$ at 80 $\mathrm{MHz}$ and $\approx 0.34 \pm 0.1$ at $40 \mathrm{MHz}$. Note that due to instrumental limitations, we observed only $|\mathrm{V}|$ with the GRIP and hence $d c p=|\mathrm{V}| / \mathrm{I}$ in the present case.

The above radio events were associated with a M1.1 class GOES soft X-ray flare during the interval $\approx 05: 46$ - 08:35 UT with peak at $\approx 06: 58 \mathrm{UT}$, a $1 \mathrm{~F}$ class $\mathrm{H} \alpha$ flare during the interval $\approx 06: 13$ - 08:33 UT with peak at $\approx 06: 37$ UT from the active region AR 11692 located at N11E12 on the solar disk2, and a 'halo' CME3. Figure 4 shows the composite of the GRAPH radioheliogram at $80 \mathrm{MHz}$, the SOHO-LASCO C2 image and the SDO-AIA $193 \AA$ A image, all obtained around $\approx$ 08:00 UT. Since the observations were during the type IVs burst period in Figure 1, the discrete radio source near the disk center is most likely the source region of the type IVs burst. Its peak brightness temperature $\left(T_{b}\right)$ is $\approx 3 \times 10^{8}$ K. Further details about the type IVs burst shall be reported elsewhere. We will limit ourselves to the type IVm burst in the rest of this paper. We would like to mention here

\footnotetext{
${ }^{2}$ swpc.noaa.gov/warehouse/2013.html

${ }^{3}$ umbra.nascom.nasa.gov/lasco/observations/halo/2013/130315
} 
that no GRAPH observations were available during the type IVm burst in Figure 1. The discrete source close to the limb in the north east quadrant in Figure 4 is presumably weak non-thermal radio noise storm activity often observed near the location of a CME in its aftermath (Kerdraon et al. 1983; Kathiravan et al. 2007). The peak $T_{b}$ of the source is $\approx 10^{7} \mathrm{~K}$

From the movies of the 'halo' CME, we find that the its leading edge (LE) was first observed in the STEREO-COR1B field of view (FOV) around $\approx 06: 15 \mathrm{UT}$ at $r \approx 1.6 R_{\odot}$. Later, at the onset time of the type IVm burst at $85 \mathrm{MHz}$ around $\approx$ 06:55 UT (Figure 1), the LE was at $r \approx 3.5 R_{\odot}$. This gives a projected linear speed of $\approx 551 \mathrm{~km} \mathrm{~s}^{-1}$ for the CME LE. The SOHO-LASCO height-time (h-t) measurements indicate that the CME LE was located at $r \approx 4.1 R_{\odot}$ around $\approx 07: 12 \mathrm{UT}^{4}$. These values are consistent with those extrapolated using the STEREO-COR1B measurements. We estimated the projected linear speed of the ejecta that moved outwards behind the CME LE observed in the STEREO-COR1B FOV (see the asterix marked feature in Figure 5). The centroid of the ejecta was at $r \approx 1.6 R_{\odot}$ during its first appearance at $\approx 06: 45 \mathrm{UT}$. Ten minutes later, i.e. at 06:55 UT close to the onset time of the type IVm burst at $85 \mathrm{MHz}$, the ejecta was at $r \approx 1.9 R_{\odot}$ (see Figure 5 ). The above h-t measurements give a speed of $\approx 348 \mathrm{~km} \mathrm{~s}^{-1}$ for the ejecta. This is nearly the same as the speed estimated using SOHO-LASCO C2 observations of the same ejecta during $\approx$ 07:24 - 08:24 UT (see Figure 4). It appears from Figures 4 and 5 that the ejecta is close to the plane of the sky for both STEREO-COR1B and SOHO-LASCO C2. The close agreement between the measured projection speeds of the CME LE and the ejecta with the above two instruments indicate that the ejecta moved at the same angle to the Sun-Earth and Sun-STEREO/B lines. As STEREO-B and Earth were separated by an angle of about $140^{\circ}$ at the time of the event, we estimate this angle to

\footnotetext{
${ }^{4}$ cdaw.gsfc.nasa.gov
} 
be $\approx 70^{\circ}$. So the angle between the ejecta and the plane of the sky for STEREO-COR1B or SOHO-LASCO C2 is $\approx 20^{\circ}$.

Any error in the position/size of the type IVs burst in Figure 4 due to propagation effects in the solar corona and the Earth's ionosphere is expected to be minimal $\left(\approx \pm 0.2 R_{\odot}\right)$ because: 1$)$ positional shifts due to refraction in the ionosphere is expected to be $\lesssim 0.2 R_{\odot}$ at $80 \mathrm{MHz}$ in the hour angle range $\pm 2 \mathrm{~h}$ (Stewart \& McLean 1982a). The local noon at Gauribidanur on 15 March 2013 was around $\approx$ 07:00 UT and the GRAPH observations described above are within the above hour angle range; 2) the effects of scattering are considered to be small at $80 \mathrm{MHz}$ compared to lower frequencies (Aubier, Leblanc \& Boischot 1971; Bastian 2004; Ramesh et al. 2006b). High angular resolution observations establishing that discrete radio sources of angular size $\approx 1^{\prime}-3^{\prime}$ are present in the solar atmosphere from where radio emission at low frequencies originate (Kerdraon 1979; Lang \& Willson 1987; Willson et al. 1998; Ramesh et al.|1999b; Ramesh \& Sastrv 2000; Ramesh \& Ebenezer 2001; Mercier et al. 2006; Kathiravan et al. 2011; Ramesh et al. 2012), ray tracing calculations indicating that the turning points of the rays that undergo irregular refraction in the solar corona nearly coincide with the location of the plasma ('critical') layer in the non-scattering case even at high frequencies like 73.8 MHz (Thejappa \& MacDowall 2008), that the maximal positional shift (for discrete solar radio sources) due to scattering is $\lesssim 0.2 R_{\odot}$ at $80 \mathrm{MHz}$ (Riddle 1974; Robinson 1983) also constrain scattering. 


\section{Results and Analysis}

\subsection{Emission mechanism}

The type IVm burst in the present case is most likely associated with the CME core like ejecta behind the CME LE because: 1) the CME LE was located at a large radial distance $\left(r \approx 3.5 R_{\odot}\right)$ at the onset time of the type IVm burst at $85 \mathrm{MHz}$. The above value is close to the outer limit of the radial distance up to which the type IVm bursts have been observed (Smerd \& Dulk 1971; Robinson 1978); 2) the location of the ejecta $\left(r \approx 1.9 R_{\odot}\right)$ close to the onset of the type IVm burst at $85 \mathrm{MHz}$ around $\approx 06: 55 \mathrm{UT}$ (see Figure 1) is consistent with the statistical estimate of the radial distance of the type IVm bursts during their onset at $80 \mathrm{MHz}$ (Smerd \& Dulk 1971); 3) no other bright moving structures were noticeable in the STEREO-COR1B FOV during $\approx$ 06:55 - 07:50 UT over $r<2 R_{\odot}$, the interval over which the radio emission was observed in Figure 1, 4) based on a statistical study of the type IVm bursts at $80 \mathrm{MHz}$, Gergely (1986) had earlier concluded that majority of the type IVm bursts move out with the ejecta behind the CME LE.

Second harmonic plasma emission and/or optically thin gyro-synchrotron emission from mildly relativistic electrons have been reported as the likely mechanisms for the type IVm bursts. Amongst the above two, we find that the latter is the cause in the present case because: 1) Wild \& Smerd (1972); Dulk (1973); Melrose (1985) had shown that the gyro-synchrotron emission is strongly suppressed at frequencies $<2 f_{p}$, where $f_{p}$ is the plasma frequency, in the presence of a medium. We estimated the total coronal electron density (i.e., the density of the background corona and the CME together) using STEREO-COR1B pB measurements at the location of the ejecta at $\approx 06: 55$ UT. The density of the background corona was determined to beginwith from a pre-CME pB image

at $\approx$ 06:05 UT using the spherically symmetric inversion method (Wang \& Davila 2014). The electron density of the ejecta was derived from the background-subtracted pB radiation 
averaged over a selected region on the ejecta (see Figure 5) and assuming the line of sight (LOS) column depth of the ejecta equal to its width for $\mathrm{pB}$ inversion. The value is $\approx 7 \times 10^{6} \mathrm{~cm}^{-3}$. This corresponds to $f_{p} \approx 24 \mathrm{MHz}$. Therefore if the type IVm burst of 2013 March 15 were because of gyro-synchrotron mechanism, emission at frequencies $\lesssim$ $48 \mathrm{MHz}$ should have been progressively weaker at $\approx 06: 55 \mathrm{UT}$. The present observations are consistent with this. The spectral profile of the type IVm burst in Figure 6) clearly shows a reduction in the observed intensity at frequencies $\lesssim 52 \mathrm{MHz}$. However at later times the burst is observable at lower frequencies (see Figure 1). This is because the ejecta had moved outward in the solar atmosphere as well as expanded in size with time (see Figures 4 and 5). As a consequence there is a gradual decrease in the total density at the location of the ejecta and hence the cut-off frequency for gyro-synchrotron emission. Probably this shift in the cut-off towards lower frequencies with time is also responsible for the observed drift of the type IVm burst in Figure 1. Note that the total density when the ejecta was first observed in the STEREO-COR1B FOV around $\approx 06: 45$ UT at $r \approx 1.6 R_{\odot}$ was $\approx 15 \times 10^{6} \mathrm{~cm}^{-3}$. Comparing this with the corresponding measurements at $\approx 06: 55$ $\mathrm{UT}$, we find that the ejecta had moved a radial distance of $\approx 0.3 R_{\odot}$ in $\approx 10 \mathrm{~min}$ and the total density during that period had decreased by about a factor of two. Note that the densities of the other rising structures (above the occulter of the coronagraph) of the CME like the 'legs' and the frontal loop which are comparitively fainter (see Figure 5) are $<7 \times 10^{6} \mathrm{~cm}^{-3}$. This indicates that the corresponding $f_{p}<24 \mathrm{MHz}$. Therefore if the type IVm burst had been due to any of the aforementioned structures of the CME, the reduction in the intensity of the burst at $\approx 06: 55 \mathrm{UT}$ should have been at lower frequencies than $\approx 52 \mathrm{MHz}$. But this is not the case; 2) the estimated $d c p$ is larger compared to that for reported for type IVm bursts due to second harmonic plasma emission (Gary et al. 1985); 3) the spectral index of the type IVm burst between $40 \mathrm{MHz}$ and $80 \mathrm{MHz}$ as estimated from the GRIP observations is $\alpha \approx-0.1$ (see Section 2 ). This is nearly the same as the 
expected spectral index for gyrosynchrotron emission over the frequency range $38.5-73.8$ MHz (Gopalswamy \& Kundu 1990).

\subsection{Magnetic field}

Dulk (1985) had shown that for optically thin non-thermal gyro-synchrotron emission, the $d c p$ and $B$ are related as follows:

$$
d c p \approx 1.26 \times 10^{0.035 \delta} 10^{-0.071 \cos \theta}\left(\frac{f}{f_{B}}\right)^{-0.782+0.545 \cos \theta}
$$

where $\theta$ is the viewing angle between the LOS and the magnetic field, $f$ is the frequency of observation, and $f_{B}=2.8 B$ is the electron gyro-frequency. The power-law index $\delta$ can be estimated from the radio flux spectral index $(\alpha)$ through the relationship $\alpha=1.20-0.90 \delta$ (Dulk 1985). In the present case $\alpha \approx-0.1$. This implies $\delta \approx 1.4$. The angle between the ejecta and the plane of the sky in the present case is $\approx 20^{\circ}$ (see Section 2 ). This indicates that the ejecta is nearly normal to the LOS and the associated field lines are likely to be radial. So we assumed the viewing angle between the LOS and the magnetic field in the type IVm burst source region to be the same as the positional angle from the LOS to the ejecta. Hence $\theta \approx 70^{\circ}$ (see Section 2 ). Substituting for the different parameters in equation (1), we get $B \approx 2.2 \pm 0.4 \mathrm{G}$ at $80 \mathrm{MHz}$ and $B \approx 1.4 \pm 0.2 \mathrm{G}$ at $40 \mathrm{MHz}$. The corresponding radial distances are most likely $r \approx 1.9 R_{\odot}(80 \mathrm{MHz})$ and $r \approx 2.2 R_{\odot}(40 \mathrm{MHz})$. We estimated this from the location of the ejecta $\left(r \approx 1.9 R_{\odot}\right)$ during the onset of the type IVm burst at $85 \mathrm{MHz}(\approx 06: 55 \mathrm{UT})$, the projected speed of the ejecta $\left(\approx 348 \mathrm{~km} \mathrm{~s}^{-1}\right)$, and the onset of the type IVm burst at $35 \mathrm{MHz}(\approx 07: 05 \mathrm{UT})$. The $d c p$ and the $B$ values are consistent with the results of the model calculations reported by Robinson (1974) for $\theta \approx 70^{\circ}$. The sense of polarization is in the e-mode. Gary et al. (1985) had remarked that gyro-synchrotron emission is a possible mechanism at $80 \mathrm{MHz}$ if conditions like $B \approx 2.8 \mathrm{G}$ at $r \approx 2.5 R_{\odot}$ are satisfied. The above estimates of $B$ at $80 \mathrm{MHz}$ in the present case agree 
reasonably with this.

\section{Summary}

A type IVm radio burst and type IVs radio burst occurred simultaneously on 2013 March 15 in association with a 'halo' CME and a M1.1/1F class soft X-ray/H $\alpha$ flare. Radio imaging, spectral and polarimeter observations of the same at low frequencies $(<$ $100 \mathrm{MHz}$ ) have been reported in this work. Our results indicate that the type IVm burst can be explained as due to optically thin gyro-synchrotron emission from the non-thermal electrons in the CME core like ejecta behind the CME LE. The estimated magnetic field strength near the type IVm burst source region is $B \approx 2.2 \pm 0.4 \mathrm{G}$ and $\approx 1.4 \pm 0.2 \mathrm{G}$ at $80 \mathrm{MHz}$ and $40 \mathrm{MHz}$, respectively. The corresponding radial distances are $r \approx 1.9 R_{\odot}(80$ $\mathrm{MHz})$ and $r \approx 2.2 R_{\odot}(40 \mathrm{MHz})$. The following results reported earlier indicate that the

above values of $B$ are plausible: 1) Dulk et al. (1976) estimated the average field strength to be in the range $\approx 3.1-0.7 \mathrm{G}$ over $r \approx 1.8-3.1 R_{\odot}$ by assuming gyro-synchrotron mechanism for similar CME associated non-thermal radio continuum; 2) Stewart et al. (1982b) reported $B>0.6 \mathrm{G}$ at $r \approx 2.5 R_{\odot}$ for the type IVm burst observed by them at $80 \mathrm{MHz}$ in association with the CME core. The authors had attributed the radio emission to be either at the fundamental or the second harmonic of the plasma frequency. Note that if the density requirements for particularly the second harmonic plasma emission are nearly the same as that for the gyro-synchrotron emission, then it is possible that the corresponding $B$ values could be similar (Dulk et al. 1976); 3) Gopalswamy \& Kundu (1989a); Bastian et al. (2001) evaluated $B \approx 1.5 \mathrm{G}$ at $r \approx 1.5 R_{\odot}$ based on similar non-thermal radio continuum due to gyro-synchrotron emission from the associated CMEs; 4) low frequency $(77 \mathrm{MHz})$ polarimeter observations of a coronal streamer associated radio source indicate that magnetic field in the former at $r \approx 1.7 R_{\odot}$ is $\approx 5 \mathrm{G}$. The present 
estimates of $B$ are reasonably consistent with this. That both the CMEs and streamers are primarily density enhancements in the solar atmosphere could be a reason for this; 5) for a similar type IVm burst event explained on the basis of optically thin gyro-synchrotron emission from the mildly relativistic non-thermal electrons in the magnetic field of the associated CME core, Tun \& Vourlidas (2013) showed that $B \approx 5-15 \mathrm{G}$ at $r \approx 1.7 R_{\odot} ; 6$ ) type IVm radio bursts associated with the 'leg' of the corresponding CMEs and generated due to second harmonic plasma emission from the enhanced electron density there indicate that $B \approx 4 \mathrm{G}$ at $r \approx 1.6 R_{\odot}$ (Ramesh et al. 2013). Considering that the coronal magnetic field associated with the active regions have a range of values (Dulk \& McLean 1978; Ramesh et al. 2003, 2011b; Sasikumar \& Ramesh 2013), the different estimates mentioned above can be regarded as reasonable. With measurements of the coronal magnetic field being very limited, particularly in close association with a CME, the results indicate that contemporaneous whitelight and radio observations of the solar corona close to the Sun $\left(r \lesssim 2 R_{\odot}\right)$ are desirable to understand the CMEs and the associated magnetic field.

It is a pleasure to thank the staff of the Gauribidanur observatory for their help in observations, maintenance of the antenna and receiver systems there. We also thank the referee for his/her comments that helped to bring out the results more clearly. The SOHO data are produced by a consortium of the Naval Research Laboratory (USA), Max-Planck-Institut fuer Aeronomie (Germany), Laboratoire d'Astronomie (France), and the University of Birmingham (UK). SOHO is a project of international cooperation between ESA and NASA. The SOHO-LASCO CME catalog and STEREO movies are generated and maintained at the CDAW Data Center by NASA and the Catholic University of America in cooperation with the Naval Research Laboratory. The SDO/AIA data are courtsey of the NASA/SDO and the AIA science teams. The work of TJW was supported by NASA Cooperative Agreement NNG11PL10A to CUA and NASA grant NNX12AB34G. 


\section{REFERENCES}

Aubier, M., Leblanc, Y., \& Boischot, A. 1971, A\&A, 12, 435

Aurass, H. Rausche, G., Mann, G., \& Hofmann, A. 2005, A\&A, 435, 1137

Bastian, T. S., \& Gary, D. E. 1997, J. Geophys. Res., 102(A7), 14031

Bastian, T. S., Pick, M., Kerdraon, A., Maia, D., \& Vourlidas, A. 2001, ApJ, 558, L65

Bastian, T. S. 2004, Planet. Sp. Sci., 52, 1381

Benz, A. O., Monstien, Ch., Meyer, H., Manoharan, P. K., Ramesh, R., et al. 2009, Earth, Moon and Planets, 104, 277

Boischot, A. 1957, C. R. Acad. Sci., 244, 1326

Boischot, A., \& Pick, M. 1962, J. Phys. Soc. Japan, 17, 203

Brueckner, G. E., et al. 1995, Sol. Phys., 162, 357

Dulk, G. A. 1973, Sol. Phys., 32, 491

Dulk, G. A., Smerd, S. F., MacQueen, R. M., Gosling, J. T., Magun, A., et al. 1976, Sol. Phys., 49, 369

Dulk, G. A., \& McLean, D. J. 1978, Sol. Phys., 57, 279

Dulk, G. A. 1985, ARA\&A, 23, 169

Duncan, R. A. 1981, Sol. Phys., 73, 191

Ebenezer, E., Ramesh, R., Subramanian, K. R., Sundara Rajan, M. S., \& Sastry, Ch. V., A\&A, 367, 1112 
Ebenezer, E., Subramanian, K. R., Ramesh, R., Sundara Rajan, M. S., \& Kathiravan, C. 2007, Bull. Astron. Soc. India, 35, 111

Gary, D. E., et al. 1985, A\&A, 152, 42

Gergely, T. E. 1986, Sol. Phys., 104, 175

Gopalswamy, N., \& Kundu, M. R. 1989a, Sol. Phys., 122, 91

Gopalswamy, N., \& Kundu, M. R. 1989b, Sol. Phys., 122, 145

Gopalswamy, N., \& Kundu, M. R. 1990, Sol. Phys., 128, 377

Grognard, R. J.-M. \& McLean, D. J. 1973, Sol. Phys., 29, 149

Howard, R. A., et al. 2008, Space Sci. Rev., 136, 67

Kai, K. 1969, Publ. Astron. Soc. Aust., 1(5), 189

Kathiravan, C., Ramesh, R., \& Nataraja, H. S. 2007, ApJ, 656, L37

Kathiravan, C., Ramesh, R., Indrajit V. Barve, \& Rajalingam, M. 2011, ApJ, 730, 91

Kerdraon, A. 1979, A\&A, 71, 266

Kerdraon, A., Pick, M., Trottet, G., Sawyer, C., Illing, R., Wagner, W., House, L. 1983, ApJ, 265, L19

Kishore, P., Kathiravan, C., Ramesh, R., Rajalingam, M., \& Indrajit V. Barve 2014, Sol. Phys., 289, 3995

Kraus J. D. 1986, Radio Astronomy, Second Edition, (Ohio: Cygnus-Quasar), 8-13

Kundu, M. R., Schmahl, E. J., Gopalswamy, N., \& White, S. M. 1989, Adv. Space Res., $9(4), 41$ 
Lang, K. R., \& Willson, R. F. 1987, ApJ, 319, 514

Lemen, J. R., et al. 2012, Sol. Phys., 275, 17L

Maia, D. J. F., Gama, R., Mercier, C., Pick, M., Kerdraon, A., Karlický, M. 2007, ApJ, 660,874

Melrose, D. B. 1985, in Solar Radio Physics, ed. D.J.McLean \& N.R.Labrum, (Cambridge: Cambridge University Press), 211

Mercier, C., et al. 2006, A\&A, 447, 1189

Pick-Gutmann, M., 1961, Ann. Astrophys., 24, 183

Pick, M. 2008, Astron. Astrophys. Rev., 16, 1

Ramesh, R., Subramanian, K. R., Sundara Rajan, M. S., \& Sastry, Ch. V. 1998, Sol. Phys., 181,439

Ramesh, R., Subramanian, K. R., \& Sastry, Ch. V. 1999a, A\&AS, 139, 179

Ramesh, R., Subramanian, K. R., \& Sastry, Ch. V. 1999b, Sol. Phys., 185, 77

Ramesh, R., \& Sastry, Ch. V. 2000, A\&A, 358, 749

Ramesh, R., \& Ebenezer, E. 2001, ApJ, 558, L141

Ramesh, R., Kathiravan, C., Sastya Narayanan, A., \& Ebenezer, E. 2003, A\&A, 400, 753

Ramesh, R., Sundara Rajan, M. S., \& Sastry, Ch. V. 2006a, Exp. Astron., 21, 31

Ramesh, R., Nataraj, H. S., Kathiravan, C., \& Sastry, Ch. V. 2006b, ApJ, 648, 707

Ramesh, R., Kathiravan, C., Sundara Rajan, M. S., Indrajit V. Barve, \& Sastry, Ch. V. 2008, Sol. Phys., 253, 319 
Ramesh, R., Kathiravan, C., \& Sastry, Ch. V. 2010, ApJ, 711, 1029

Ramesh, R. 2011a, in Proc. of the 1st Asia-Pacific Solar Physics Meeting, ed. A.R.Choudhuri \& D.Banerjee (Astron. Soc. India Conf. Ser.), 2, 55

Ramesh, R., Kathiravan, C., \& Satya Narayanan, A. 2011b, ApJ, 734, 39

Ramesh, R., Kathiravan, C., Indrajit V. Barve, \& Rajalingam, M. 2012, ApJ, 744, 165

Ramesh, R., Kishore, P., Sargam M. Mulay, Indrajit V. Barve, Rajalingam, M., Kathiravan, C., \& Wang, T. J. 2013, ApJ, 778, 30

Riddle, A. C. 1974, Sol. Phys., 35, 153

Robinson, R. D. 1974, Publ. Astron. Soc. Aust., 2(5), 258

Robinson, R. D. 1978, Sol. Phys., 60, 383

Robinson, R. D. 1983, Proc. Astron. Soc. Aust., 5, 208

Sasikumar Raja, K., \& Ramesh, R. 2013, ApJ, 775, 38

Smerd, S. F., \& Dulk, G. A. 1971, in Solar Magnetic Fields, ed. R. Howard, (Dordrecht: Reidel), IAU Symp. 43, 616

Stewart, R. T., \& McLean, D. J. 1982a, Proc. Astron. Soc. Aust., 4(4), 386

Stewart, R. T., et al. 1982b, A\&A, 116, 217

Stewart, R. T. 1985, in Solar Radio Physics, ed. D.J.McLean \& N.R.Labrum, (Cambridge: Cambridge University Press), 361

Subramanian, K. R., \& Sastry, Ch. V. 1988, J. Astrophys. Astron., 9, 225

Thejappa, G., \& MacDowall, R. J. 2008, ApJ, 676, 1338 
Tun, S. D., \& Vourlidas, A. 2013, ApJ, 766, 130

Wang, T. J., \& Davila, J. M. 2014, Sol. Phys., 289, 3723

Weiss, A. A. 1963, Aust. J., Phys., 16, 526

White, S. M. 2007, Asian J. Phys., 16, 189

Wild, J. P., Smerd, S. F., \& Weiss, A. A. 1963, ARA\&A, 1, 291

Wild, J. P., \& Smerd, S. F. 1972, ARA\&A, 10, 159

Willson, R. F., Redfield, S. L., Lang, K. R., Thompson, B. J., \& St. Cyr., O. C. 1998, ApJ, 504, L117 


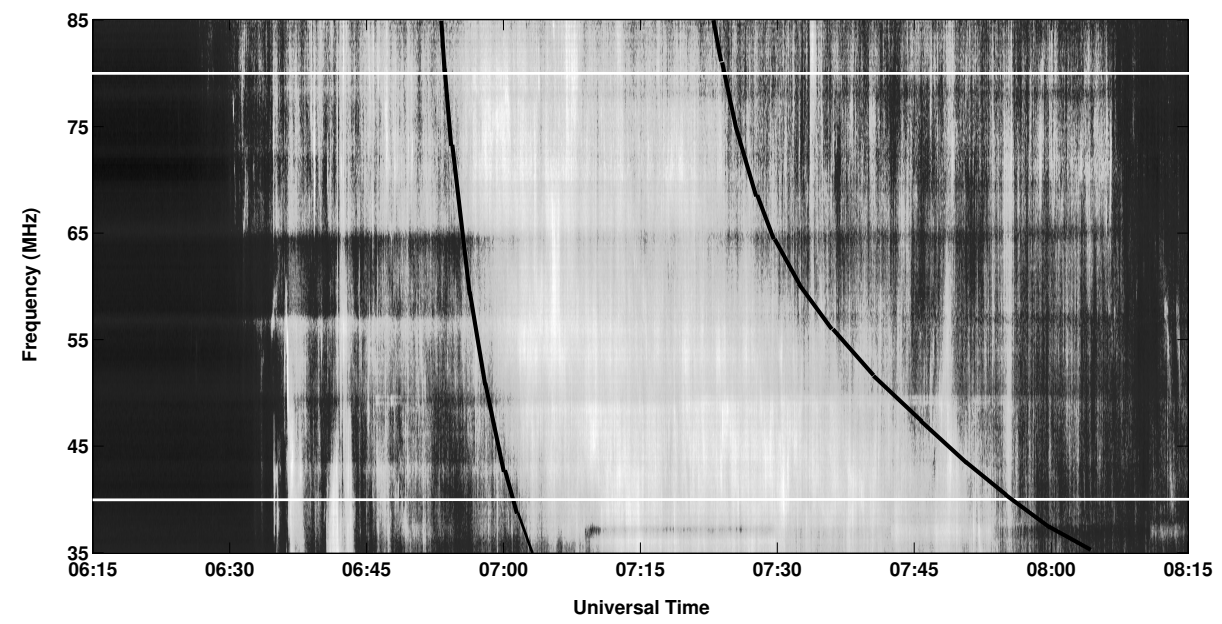

Fig. 1. - Dynamic spectrum of the solar radio emission observed with the GLOSS on 2013 March 15 during 06:15 - 08:15 UT in the frequency range 85 - 35 MHz. The stationary emission during the interval $\approx 06: 30$ - 08:10 UT and the drifting emission during $\approx 06: 55$ 07:50 UT correspond respectively to the type IVs and type IVm bursts mentioned in the text. The two 'white' horizontal lines indicate $40 \mathrm{MHz}$ and $80 \mathrm{MHz}$ portion of the spectrum. The other horizontal line like features noticeable in the spectrum, for eg. near $\approx 55 \mathrm{MHz} \approx 65$ $\mathrm{MHz}$, etc. are due to local radio frequency interference (RFI). The two slanted 'black' lines indicate the approximate interval over which the type IVm burst was observed at different frequencies. 


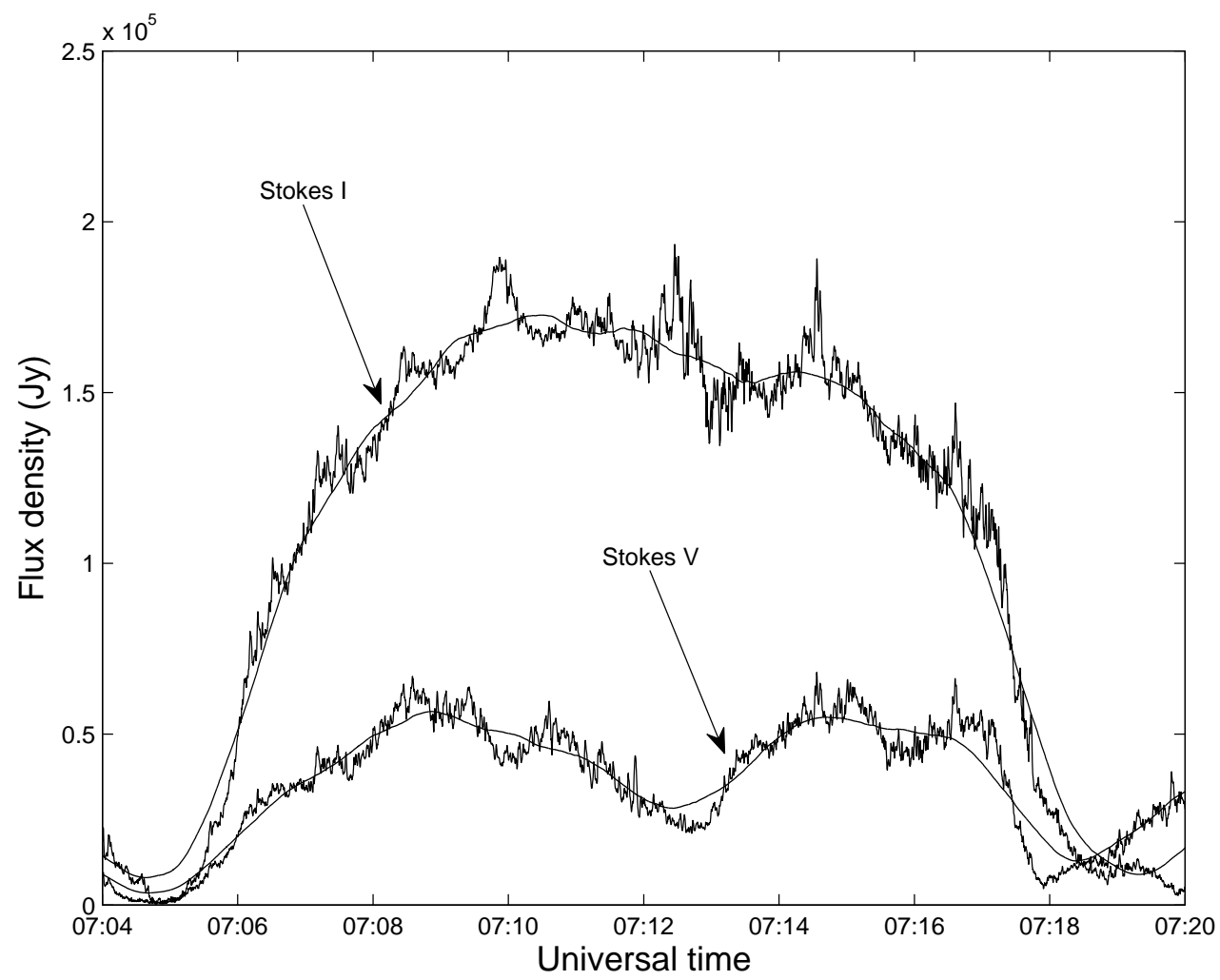

Fig. 2.- Time profile of the Stokes I and Stokes V emission observed with the GRIP at 80 MHz on 2013 March 15 in the transit mode. The duration of the observations correspond approximately to the width of the response pattern of the GRIP at $80 \mathrm{MHz}$ in the eastwest direction (see Section 2). The overplotted smooth line is the polynomial fit to the observations. 


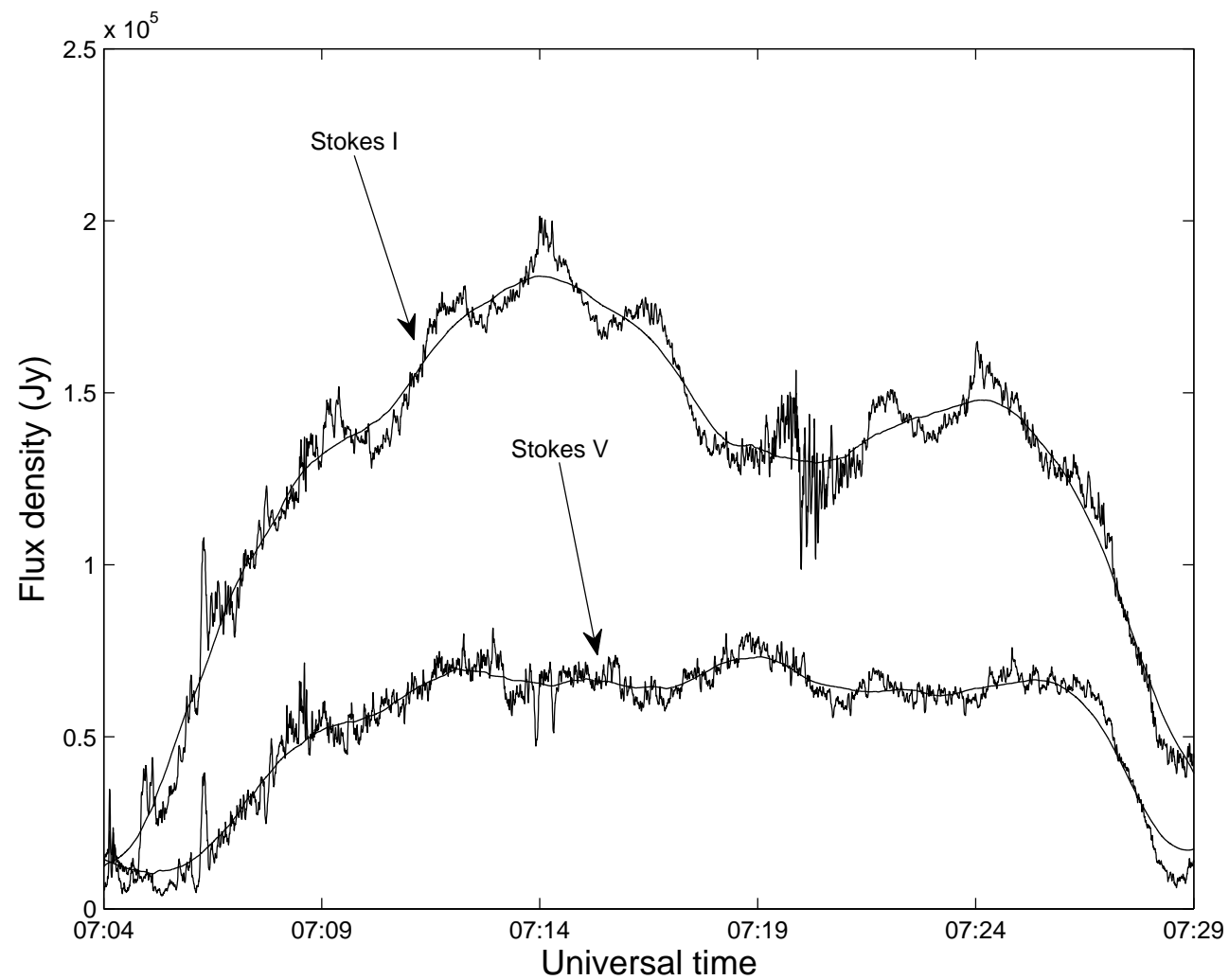

Fig. 3.- Same as Figure 2 but at $40 \mathrm{MHz}$. 


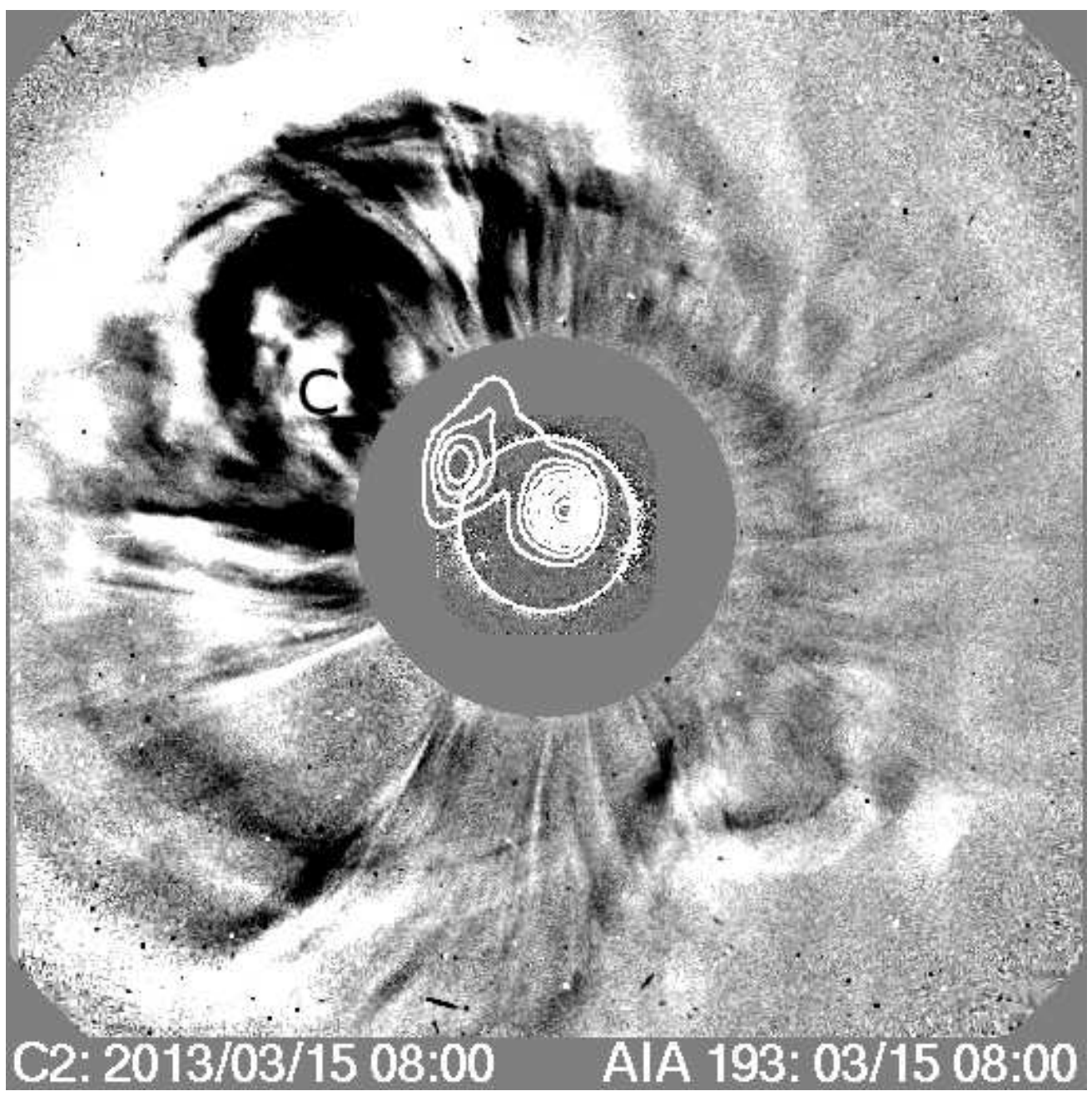

Fig. 4.- A composite of the $80 \mathrm{MHz}$ GRAPH radioheliogram of the type IVs burst observed on 2013 March 15 around $\approx$ 08:00 UT (contours in white colour) and the SOHO-LASCO C2, SDO-AIA (193 $\AA$ ) images obtained close to the same time that day. The discrete source of radio emission near the disk center is the type IVs burst mentioned in the text. The 'white' circle(radius $\left.=1 R_{\odot}\right)$ at the center indicates the solar limb. The bigger, concentric 'grey' circle (radius $\approx 2.2 R_{\odot}$ ) represents the occulting disk of the SOHO-LASCO C2 coronagraph. Solar north is straight up and solar east is to the left in the image. The white light feature marked ' $\mathrm{C}$ ' is the CME core like ejecta mentioned in the text. 


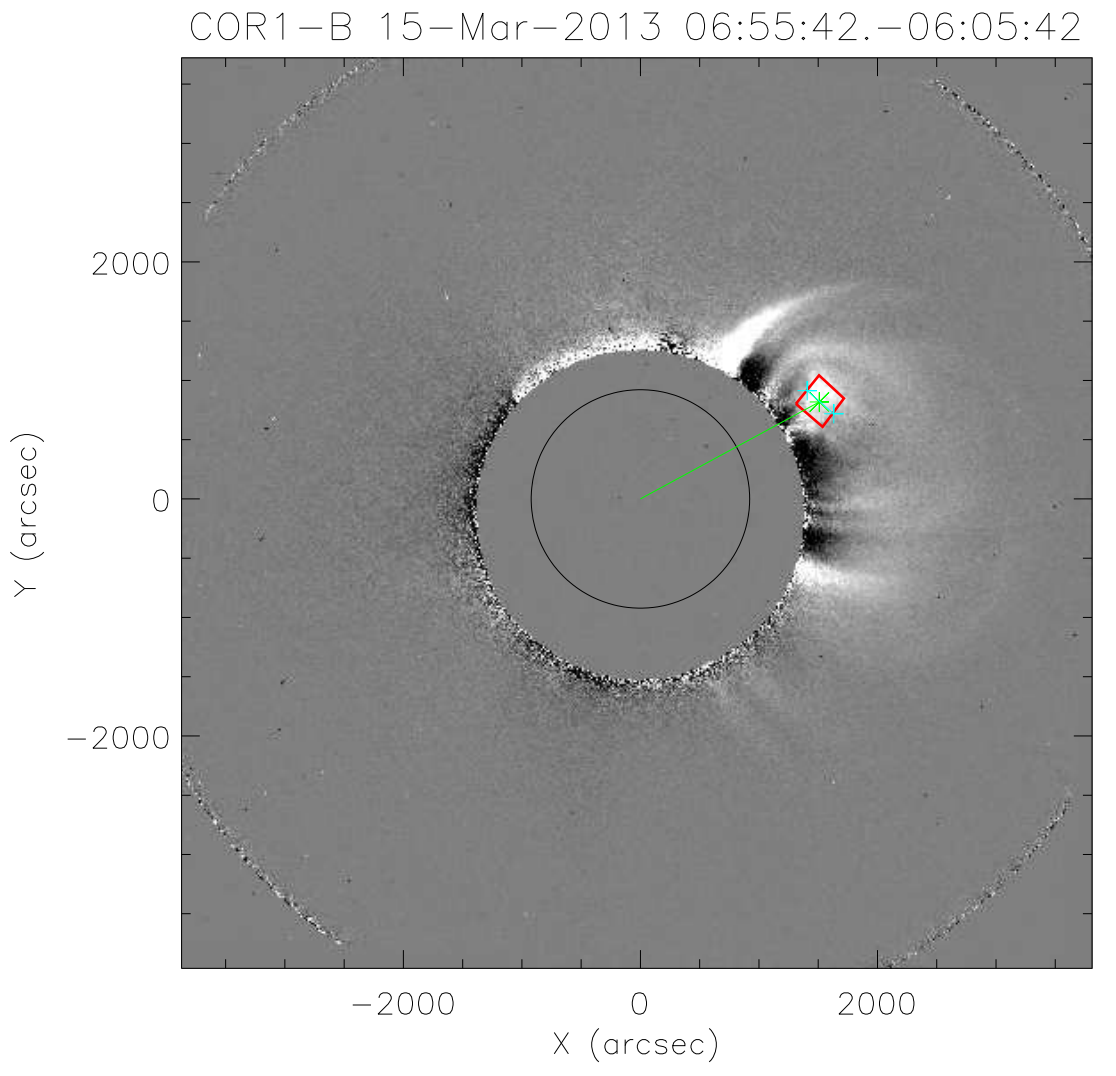

Fig. 5. - STEREO-COR1B pB difference image obtained on 2013 March 15 around 06:55 UT. The subtracted reference image was observed at 06:05 UT prior to the CME onset. The region marked with a rectangular box is used for measuring the density of the CME ejecta. The 'grey' circle (radius $\approx 1.4 R_{\odot}$ ) represents the occulting disk of the coronagraph. The asterisk within the rectangular box marks the same feature (ejecta) marked 'C' in Figure 4 . 


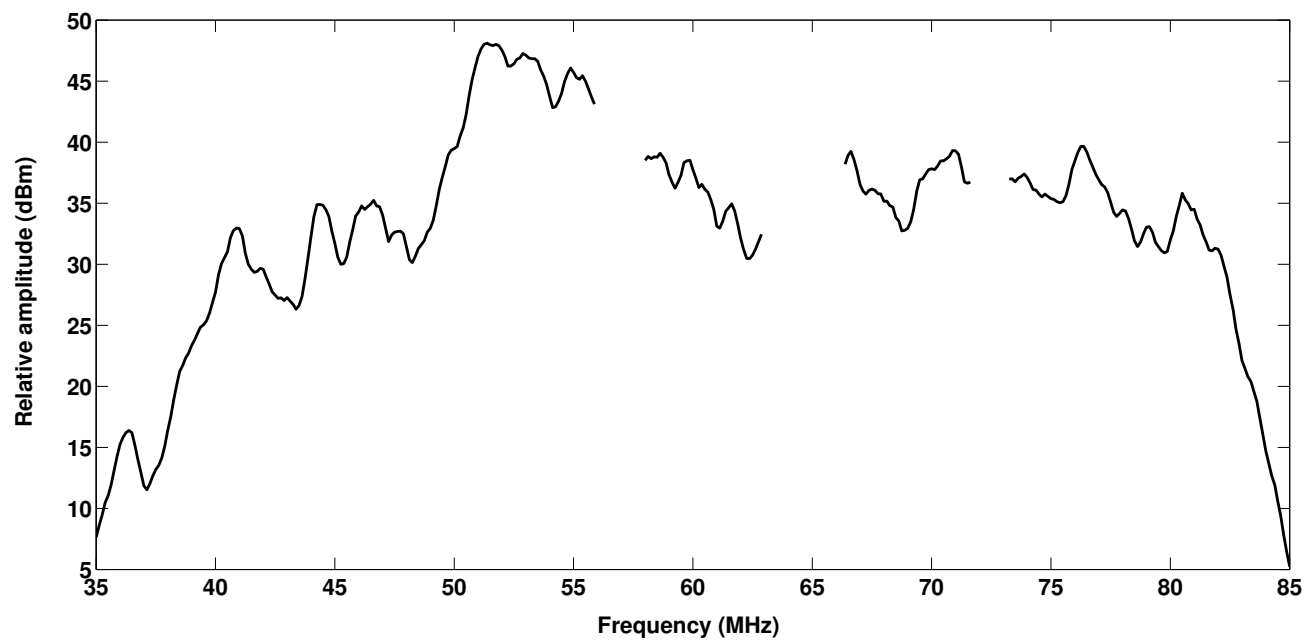

Fig. 6. - Spectral profile of the type IVm burst in Figure 1 at $\approx 06: 55$ UT. The gaps in the profile near $\approx 55 \mathrm{MHz}, \approx 65 \mathrm{MHz}$, etc. correspond to the frequency channels affected by RFI. 